\title{
Correction to: A repeated cross-sectional study of clinicians' use of psychotherapy techniques during 5 years of a system-wide effort to implement evidence-based practices in Philadelphia
}

Rinad S. Beidas ${ }^{1,2,3^{*}}$, Nathaniel J. Williams ${ }^{4}$, Emily M. Becker-Haimes ${ }^{1,15}$, Gregory A. Aarons ${ }^{5}$, Frances K. Barg ${ }^{6}$, Arthur C. Evans ${ }^{7}$, Kamilah Jackson ${ }^{8}$, David Jones ${ }^{9}$, Trevor Hadley ${ }^{1}$, Kimberly Hoagwood ${ }^{10}$, Steven C. Marcus ${ }^{11}$, Geoffrey Neimark ${ }^{8}$, Ronnie M. Rubin ${ }^{8}$, Sonja K. Schoenwald ${ }^{12}$, Danielle R. Adams ${ }^{13}$, Lucia M. Walsh ${ }^{14}$, Kelly Zentgraf ${ }^{1}$ and David S. Mandell ${ }^{1,3}$

\section{Correction to: Beidas et al. Implement Sci (2019) 14:67 https://doi.org/10.1186/s13012-019-0912-4}

Following publication of the original article [1], the authors reported an error in the "Participants" subsection. The original article states:

Over the course of the 5 years, we enrolled 21 out of the 29 organizations (73\%; some organizations had more than one site, resulting in a total of 27 sites).

However, it should state:

Over the course of the 5 years, we enrolled 22 out of the 29 organizations $(\mathbf{7 6 \%}$; some organizations had more than one site, resulting in a total of $\mathbf{3 1}$ sites).

\footnotetext{
Author details

'Department of Psychiatry, Perelman School of Medicine, University of Pennsylvania, Philadelphia, PA, USA. ${ }^{2}$ Department of Medical Ethics and Health Policy, Perelman School of Medicine, University of Pennsylvania, Philadelphia, PA, USA. ${ }^{3}$ Penn Implementation Science Center at the Leonard Davis Institute of Health Economics (PISCE@LDI), University of Pennsylvania, Hall- Mercer Community Mental Health Center, Philadelphia, PA, USA. ${ }^{4}$ School of Social Work, Boise State University, Boise, ID, USA. ${ }^{5}$ Department of Psychiatry, University of California San Diego, San Diego, CA, USA.

${ }^{6}$ Department of Family Medicine and Community Health, University of Pennsylvania Perelman School of Medicine, Philadelphia, PA, USA. ${ }^{7}$ American Psychological Association, Washington, DC, USA. ${ }^{8}$ Community Behavioral Health, Impact Reach, LLC, Philadelphia, PA, USA. ${ }^{9}$ Department of Behavioral Health, Philadelphia, PA, USA. ${ }^{10}$ Department of Child and Adolescent Psychiatry, New York University Langone Health, New York, NY, USA. " 5 School
}

of Social Policy and Practice, University of Pennsylvania, Philadelphia, PA, USA. ${ }^{12}$ Oregon Social Learning Center, Eugene, OR, USA. ${ }^{13}$ School of Social Service Administration, University of Chicago, Chicago, IL, USA. ${ }^{14}$ Department of Psychology, University of Miami, Miami, FL, USA. ${ }^{15}$ Hall- Mercer

Community Mental Health Center, $16 \mathrm{lmpact}$ Reach, LLC, Philadelphia, PA, USA.

Published online: 09 September 2019

\section{Reference}

1. Beidas RS, et al. A repeated cross-sectional study of clinicians' use of psychotherapy techniques during 5 years of a system-wide effort to implement evidence-based practices in Philadelphia. Implement Sci. 2019; 14:67. https://doi.org/10.1186/s13012-019-0912-4.

* Correspondence: rbeidas@upenn.edu

1 Department of Psychiatry, Perelman School of Medicine, University of Pennsylvania, Philadelphia, PA, USA

${ }^{2}$ Department of Medical Ethics and Health Policy, Perelman School of

Medicine, University of Pennsylvania, Philadelphia, PA, USA

Full list of author information is available at the end of the article

(c) The Author(s). 2019 Open Access This article is distributed under the terms of the Creative Commons Attribution 4.0 International License (http://creativecommons.org/licenses/by/4.0/), which permits unrestricted use, distribution, and reproduction in any medium, provided you give appropriate credit to the original author(s) and the source, provide a link to the Creative Commons license, and indicate if changes were made. The Creative Commons Public Domain Dedication waiver (http://creativecommons.org/publicdomain/zero/1.0/) applies to the data made available in this article, unless otherwise stated. 